\title{
Geometric coupling thresholds in a two-dimensional strip
}

\author{
D. Borisov ${ }^{a}$, P. Exner, ${ }^{b, c}$ and R. Gadyl'shin ${ }^{a, d}$
}

a) Bashkir State Pedagogical University, October Revolution St. 3a, 450000 Ufa, Russia

b) Nuclear Physics Institute, Academy of Sciences, 25068 Řez near Prague, Czechia

c) Doppler Institute, Czech Technical University, Břehová 7, 11519 Prague, Czechia

d) Institute of Mathematics, Ufa Science Center, Russian Academy of Sciences, Chernyshevskogo St., 112, 450000, Ufa, Russia

BorisovDI@ic.bashedu.ru, exner@ujf.cas.cz, gadylshin@bspu.ru

We consider the Laplacian in a strip $\mathbb{R} \times(0, d)$ with the boundary condition which is Dirichlet except at the segment of a length $2 a$ of one of the boundaries where it is switched to Neumann. This operator is known to have a non-empty and simple discrete spectrum for any $a>0$. There is a sequence $0<a_{1}<a_{2}<\cdots$ of critical values at which new eigenvalues emerge from the continuum when the Neumann window expands. We find the asymptotic behavior of these eigenvalues around the thresholds showing that the gap is in the leading order proportional to $\left(a-a_{n}\right)^{2}$ with an explicit coefficient expressed in terms of the corresponding threshold-energy resonance eigenfunction. 


\section{Introduction}

Spectra of Dirichlet Laplacians in infinitely stretched regions such as planar strips or layers with local perturbations were studied recently in numerous papers. The motivation for this work came from applications in condensed matter physics, and also from the fact that it was itself an interesting mathematical problem.

One of the simplest systems of this type is a free quantum particle confined to a pair of straight parallel strips with Dirichlet boundary conditions coupled laterally by a window in the common boundary. If they are of the same width $d$, one can employ the mirror symmetry and concentrate on the nontrivial part which is equivalent to the analysis of the Laplacian in a single strip with the Dirichlet boundary condition switched to Neumann at a finite segment of one of the boundaries.

Such a system has at least one bound state for any "window" length $2 a>0$ as it was found in [ESTV] and independently in [BGRS]. The discrete spectrum is simple, the eigenvalues $\lambda_{n}, n=0,1, \ldots$, are continuously decreasing as functions of $a$ and their number is linear in $a$ up to an error term as the window is widening [ESTV]. These properties follow from a simple bracketing argument which allows to squeeze the eigenvalues between those of a box covering the "coupled" part with Dirichlet and Neumann conditions at $x_{1}= \pm a$. It shows, in particular, that there are critical values $a_{n}, n=0,1, \ldots$, at which new eigenvalues emerge from the continuum. By [ESTT, BGRS] we have $a_{0}=0$ while generally we know only that

$$
a_{n} \in\left(\frac{n d}{\sqrt{3}}, \frac{(n+1) d}{\sqrt{3}}\right), \quad n=1,2, \ldots
$$

This tells us nothing about the behavior of the eigenvalues around the critical points.

The weak coupling asymptotics was studied for the ground state. A variational method of [EV yields for small $a$ a two-sided estimate of $\lambda_{0}(a)$ between two multiples of $a^{4}$. This is indeed the leading term: using the matching method of [1], Ga1 Popov derived in $\mathrm{Po}$ the 
expansion

$$
\lambda_{0}(a)=\left(\frac{\pi}{d}\right)^{2}-\left(\frac{\pi^{3}}{2 d^{3}}\right)^{2} a^{4}+\mathcal{O}\left(a^{5}\right) .
$$

While his argument is not fully rigorous because an estimate of the error term is missing, the formula itself raises no doubts, in particular, because of its excellent agreement with the numerical result of [EŠTV]. The result is subtle: recall that (1.2) differs substantially from the asymptotics corresponding to a local change in mixed boundary conditions, where the Birman-Schwinger technique is applicable and the leading term is a multiple of the window width squared [EK].

In the present paper we address the question about the behavior of the higher eigenvalues $\lambda_{n}, n=1,2, \ldots$, in the vicinity of the critical points $a_{n}$. It is a natural counterpart of the coupling constant threshold problem of [KS]. Our main result is that the gap between an eigenvalue $\lambda_{n}$ and the continuum is proportional to $\left(a-a_{n}\right)^{2}$ with a coefficient given explicitly in terms of the corresponding thresholdenergy resonance eigenfunction. This fits well into the analogy between our problem and spectral properties of one-dimensional Schrödinger operators. The latter extends to higher dimensions, but the argument becomes more complicated and we leave that to another paper.

To finish the introduction, let us say something about the method. We deal with a perturbative problem with respect to the parameter $\varepsilon$ defined as the excess of the window halfwidth $a$ over the critical value $a_{n}$. Since the latter is positive for $n \geq 1$, the problem in question is a regular one. This fact makes it possible to map the problem into an equivalent one with a small and local perturbation of the equation and the boundary condition fixed, i.e. independent of $\varepsilon$. This is what we are going to do. We employ the technique introduced in Ga2 for calculations of the eigenfunctions for one-dimensional perturbed Schrödinger operator; its advantage is that we arrive at the sought asymptotic formula in a straightforward and reasonably simple way. 


\section{The main result}

To formulate the result we need first to introduce some notation and recall a few simple facts about the problem in question. We employ Cartesian coordinates, $x=\left(x_{1}, x_{2}\right)$, in which $\Sigma=\left\{x: 0<x_{2}<d\right\}$. The upper strip boundary is $\Gamma=\left\{x: x_{2}=d\right\}$, while the lower decomposes into the union of $\gamma^{a}=\left\{x:\left|x_{1}\right|<a, x_{2}=0\right\}$ and $\Gamma^{a}=\left\{x:\left|x_{1}\right|>a, x_{2}=0\right\}$. The operator $H_{a}$ we are going to consider is the Laplacian, $H_{a} \psi=-\Delta \psi$, in $L^{2}(\Sigma)$ with the Dirichlet boundary condition on $\Gamma^{a} \cup \Gamma$ and Neumann on $\gamma^{a}$; it is a well-defined self-adjoint operator - cf. [Da, Chap. 7], [DK].

Proposition 2.1 The discrete part of $\sigma\left(H_{a}\right)$ is simple and the eigenvalues $\lambda_{n}(a):\left(\frac{\pi}{2 d}\right)^{2}<\lambda_{0}(a)<\lambda_{1}(a)<\cdots<\left(\frac{\pi}{d}\right)^{2}$ are continuous and monotonously decreasing with respect to a. There are numbers $0=a_{0}<a_{1}<a_{2}<\cdots$ satisfying (1.1) such that

(a) for $a \in\left(a_{n-1}, a_{n}\right]$ the operator $H_{a}$ has exactly $n$ eigenvalues,

(b) for $a>a_{n}$ we denote $\varepsilon:=a-a_{n}$, then the eigenfunction $\psi_{n}^{(\varepsilon)}$ associated with $\lambda_{n}(a)$ has a definite parity with respect to $x_{1}$, namely

$$
\psi_{n}^{(\varepsilon)}\left(-x_{1}, x_{2}\right)=(-1)^{n} \psi_{n}^{(\varepsilon)}\left(x_{1}, x_{2}\right),
$$

(c) for $a=a_{n}$ the equation $-\Delta \psi=\left(\frac{\pi}{d}\right)^{2} \psi$ with given boundary conditions has a solution $\psi_{n}^{(0)} \in H_{\mathrm{loc}}^{1}(\Sigma)$, unique up to a multiplicative constant, which behaves like

$$
\psi_{n}^{(0)}(x)=c_{1}( \pm 1)^{n} \sin \left(\frac{\pi x_{2}}{d}\right)+\mathcal{O}\left(\mathrm{e}^{-\delta\left|x_{1}\right|}\right)
$$

as $x_{1} \rightarrow \pm \infty$, where $\delta:=\pi \sqrt{3} / d$ (in what follows we set $c_{1}:=\sqrt{2 / \pi}$ for the sake of definiteness).

Proof: Most part was demonstrated in ESTV], it remains to check the claim (c). Any solution can be expanded in terms of the transverse eigenfunction bases

$$
\chi_{j}\left(x_{2}\right):=\sqrt{\frac{2}{d}} \sin \left(\frac{\pi j x_{2}}{d}\right), \phi_{j}\left(x_{2}\right):=\sqrt{\frac{2}{d}} \sin \left(\frac{\pi(2 j-1)\left(d-x_{2}\right)}{d}\right)
$$


with $j=1,2, \ldots$. Since the problem has a mirror symmetry with respect to $x_{1}=0$, it is sufficient to discuss the halfstrip part, $x_{1} \geq 0$, with the appropriate boundary condition at $x_{1}=0$. Let is consider the even case. A solution of energy $\epsilon\left(\frac{\pi}{d}\right)^{2}$ expresses as

$$
\psi(x)=\sum_{j=1}^{\infty} c_{j} \mathrm{e}^{q_{j}\left(a-x_{1}\right)} \chi_{j}\left(x_{2}\right), \quad \psi(x)=\sum_{j=1}^{\infty} b_{j} \frac{\cosh \left(p_{j} x_{1}\right)}{\cosh \left(p_{j} a\right)} \phi_{j}\left(x_{2}\right)
$$

for $\left|x_{1}\right| \geq a$ and $\left|x_{1}\right| \leq a$, respectively, where $q_{j}:=\frac{\pi}{d} \sqrt{j^{2}-\epsilon}$ and $p_{j}:=$ $\frac{\pi}{d} \sqrt{\left(j-\frac{1}{2}\right)^{2}-\epsilon}$. The coefficients in the above relation are determined by the requirement of smoothness of $\psi$ at the segment $x_{1}=a$; we have

$$
c_{j}=\sum_{k=1}^{\infty} b_{k}\left(\chi_{j}, \phi_{k}\right), \quad\left(\chi_{j}, \phi_{k}\right)=\frac{(-1)^{j-k}}{\pi} \frac{2 j}{j^{2}-\left(k-\frac{1}{2}\right)^{2}},
$$

and $b=\left\{b_{j}\right\}$ is given as solution of an infinite system of equations which can be written concisely in the operator form

$$
C b=0
$$

with

$$
C_{j k}:=\left[q_{j}+p_{k} \tanh \left(p_{k} a\right)\right]\left(\chi_{j}, \phi_{k}\right) .
$$

The odd case is similar, just cosh and tanh are replaced by sinh and coth, respectively. The allowed values of $\epsilon$ are those for which a solution to the system (2.4) exists.

We know from [ESTV that the sequence corresponding to an isolated eigenvalue of $H_{a}$, i.e. $\epsilon \in\left(\frac{1}{4}, 1\right)$, belongs to $\ell^{2}\left(j^{-r}\right)$ for any $r \geq 1$, and that $C=C(a, \epsilon)$ is Hilbert-Schmidt on $\ell^{2}\left(j^{-r}\right)$ with $r$ large enough independently of $a$ and $\epsilon$. Choosing such an $r$ it is straightforward to check that $(a, \epsilon) \mapsto C(a, \epsilon)$ is jointly continuous in the corresponding Hilbert-Schmidt norm. Take $a>a_{n}$ and $\epsilon_{n}(a):=\left(\frac{d}{\pi}\right)^{2} \lambda_{n}(a)$, clearly $\epsilon_{n}(a) \rightarrow 1$ as $a \rightarrow a_{n}+$. The said continuity implies that the equation (2.4) has for $C\left(a_{n}, 1\right)$ a unique solution in $\ell^{2}\left(j^{-r}\right)$, and by (2.3) it determines a sequence $b \in \ell^{2}\left(j^{-r}\right)$. Together they yield the sought 
threshold resonance solution for $a=a_{n}$ with the asymptotics (2.1) following from the first one of the relations (2.2).

Remark 2.2 The function $\psi_{n}^{(0)}$ described in Proposition 2.1 has the following smoothness properties. It is infinitely differentiable everywhere in $\bar{\Sigma}$ except the endpoints of the segment $\gamma^{a_{n}}$. At these points the asymptotic formula

$$
\psi_{n}^{(0)}(x)=( \pm 1)^{n} \alpha_{n} r_{ \pm}^{1 / 2} \sin \frac{\theta_{ \pm}}{2}+\mathcal{O}\left(r_{ \pm}\right), \quad r_{ \pm} \rightarrow 0,
$$

is valid, where $\left(r_{ \pm}, \theta_{ \pm}\right)$are polar coordinates associated with the variables $\left( \pm x_{1}-a_{n}, x_{2}\right)$ and $\alpha_{n}$ is a some number (a unique one provided we fix $c_{1}$ ). These asymptotics formulas can be verified in two easy steps. First, one should extend the function $\psi_{n}^{(0)}$ to the mirrored strip $\left\{x:-d<x_{2}<0\right\}$ in the even way. This leads to solution of the equation $-\Delta \psi_{n}^{(0)}=\left(\frac{\pi}{d}\right)^{2} \psi_{n}^{(0)}$ in the double strip $\left\{x:\left|x_{2}\right|<d\right\} \backslash\{x$ : $\left.\left|x_{1}\right|>a, x_{2}=0\right\}$ with the Dirichlet boundary condition at the outer boundary and at the cut $\left\{x:\left|x_{1}\right|>a, x_{2}= \pm 0\right\}$, and it is sufficient to employ the results established in [NP] for such elliptic problems.

Now we can state our main result:

Theorem 2.3 The eigenvalue $\lambda_{n}(a)$ of $H_{a}$ with $n \geq 1$ has the following asymptotic behavior,

$$
\lambda_{n}(a)=\left(\frac{\pi}{d}\right)^{2}-\mu_{n}^{2}\left(a-a_{n}\right)^{2}+\mathcal{O}\left(\left(a-a_{n}\right)^{3}\right)
$$

as $a \rightarrow a_{n}+$, where the coefficient is given by

$$
\mu_{n}:=\frac{1}{a_{n}} \int_{\Sigma}\left|\frac{\partial \psi_{n}^{(0)}}{\partial x_{1}}\right|^{2} d x_{1} d x_{2},
$$

or alternatively by

$$
\mu_{n}:=\frac{\pi \alpha_{n}^{2}}{4}
$$


where $\alpha_{n}$ is the number appearing in (2.5). The associated eigenfunction $\psi_{n}^{(\varepsilon)}$ can be normalized in such a way that it satisfies the relation

$$
\psi_{n}^{(\varepsilon)}=\psi_{n}^{(0)}+\mathcal{O}(\varepsilon),
$$

in $H^{1}((-R, R) \times(0, d))$ for any $R>0$ behaving asymptotically as

$$
\psi_{n}^{(\varepsilon)}(x)=c_{1}( \pm 1)^{n} \mathrm{e}^{-\varepsilon \mu_{n}\left|x_{1}\right|} \sin \left(\frac{\pi x_{2}}{d}\right)+\mathcal{O}\left(\mathrm{e}^{-\delta\left|x_{1}\right|}\right)
$$

when $x_{1} \rightarrow \pm \infty$, with $\delta$ defined in Proposition 2.1.

Remark 2.4 The function $\psi_{n}^{(\varepsilon)}$ belongs, of course, to $L^{2}(\Sigma)$, but it does not have a limit in this space as $\varepsilon \rightarrow 0+$ since the norm $\left\|\psi_{n}^{(\varepsilon)}\right\|_{L^{2}(\Sigma)}$ explodes in the limit. Furthermore, $\alpha_{n}$ is nonzero for any $n \geq 1$. This fact can be easily deduced from the assertion (2.7). Indeed, the assumption $\alpha_{n}=0$ implies immediately that $\psi_{n}^{(0)}$ is independent on $x_{1}$. However, this contradicts to the boundary value problems that $\psi_{n}^{(0)}$ satisfies to. The coefficient $\alpha_{n}$ being nonzero, the formula (2.8) shows that the asymptotics of each $\lambda_{n}$ is nontrivial in the leading order.

\section{Proof of Theorem 2.2}

The rest of the paper is devoted to the proof of Theorem 2.3. With the scaling behavior of the spectrum in mind we can put $d=\pi$ without loss of generality in the following. Furthermore, the mirror symmetry with respect to $x_{1}=0$ makes it possible to consider the halfstrip problem with the Dirichlet or Neumann condition at the cut.

We need to introduce some notations. Let $\mathbb{R}_{+}^{2}=\left\{x: x_{1}>0\right\}$ be the open right halfplane. As indicated above, we will work in the halfstrip $\Pi:=\Sigma \cap \mathbb{R}_{+}^{2}$, similarly we introduce $\gamma_{\varepsilon}:=\gamma^{a_{n}+\varepsilon} \cap \mathbb{R}_{+}^{2}, \Gamma_{+}:=$ $\Gamma \cap \mathbb{R}_{+}^{2}$, and $\Gamma_{\varepsilon}:=\Gamma^{a_{n}+\varepsilon} \cap \mathbb{R}_{+}^{2}$. Moreover, we need a symbol for the cut at the symmetry axis, $\gamma:=\left\{x: x_{1}=0,0<x_{2}<\pi\right\}$. Since we consider a fixed eigenvalue, we shall omit the index $n$ when there is no danger of misunderstanding. 
Let us outline the strategy of the proof. In the first step we are going to analyze the problem

$$
\begin{aligned}
(\Delta+1) u & =m^{2} u+f, \quad x \in \Pi, \\
l_{x} u & =0, \quad x \in \gamma, \\
u & =0, \quad x \in \Gamma_{0} \cup \Gamma_{+}, \\
\frac{\partial u}{\partial x_{2}} & =0, \quad x \in \gamma_{0},
\end{aligned}
$$

with a fixed function $f$ at the right-hand side of the equation. The trace operator $l_{x}$ in the boundary conditions is defined as $\left(l_{x} u\right)\left(x_{1}, x_{2}\right)=$ $u\left(0, x_{2}\right)$ if $n$ is odd and $\left(l_{x} u\right)\left(x_{1}, x_{2}\right)=\frac{\partial u}{\partial x_{1}}\left(0, x_{2}\right)$ if $n$ is even. The parameter $m$ is assumed to be complex and to lie in a (sufficiently small) neighborhood of zero (we indicate this neighborhood by $\mathcal{D}$ ). We also suppose that $f$ is an arbitrary function from $L^{2}(\Pi)$ with a compact support. We will construct a solution of the problem (3.1), meromorphic with respect to $m$, with the following asymptotic behavior far from the cut of the halfstrip,

$$
u(x, m)=c(m) \mathrm{e}^{-m x_{1}} \sin x_{2}+\mathcal{O}\left(\mathrm{e}^{-\sqrt{3+m^{2}} x_{1}}\right), \quad x_{1} \rightarrow+\infty,
$$

where $c(m)$ is a constant determined by the function $f$. In the second step we will transform the original boundary value problem for the eigenfunction $\psi^{(\varepsilon)}$ to another one with an equation the coefficients of which depend smoothly on $\varepsilon$ and the boundary condition is independent of $\varepsilon$. What is important is that the reformulated problem will be of the form (3.1) with a particular right-hand side $f=f_{\varepsilon}$ for which we deduce a sufficient explicit representation. Combining the latter with properties of the solution to (3.1) will finally obtain the announced

results concerning $\lambda_{\varepsilon}$ and $\psi^{(\varepsilon)}$. Since the proof of Theorem 2.3 divides in this way naturally into two steps, we shall discuss them separately in the two following subsections.

\subsection{Solution of the problem (3.1)}

As we have indicated our aim is to construct a solution of (3.1) which is meromorphic in $m \in \mathcal{D}$. Let us say more explicitly what we mean 
by that. It is easy to see that there is a unique solution for $m \in$ $\mathcal{D} \cap\{m: \operatorname{Re} m>0\}$ which decays as $x_{1} \rightarrow \infty$. We shall check that as a function of $m$ it is analytic in $\mathcal{D} \cap\{m: \operatorname{Re} m>0\}$ and extend it to the remaining part of $\mathcal{D}$. The extension will be for $\operatorname{Re} m<0$ again a solution to (3.1) with the asymptotics (3.2), and in addition, it will be meromorphic in $m \in \mathcal{D}$ with just one simple pole at $m=0$. Of course, the extension will be bounded at large distances if $\operatorname{Re} m=0$ and will be increasing for $\operatorname{Re} m<0$. Speaking about solutions everywhere in the following we mean always such analytic continuations.

Recall that a function $F$ with values in some Banach space $X$ is said to be holomorphic in $\mathcal{D}$ if it is differentiable (in the sense of the norm of $X$ ) at each point $m \in \mathcal{D}$. If this function is holomorphic in $\mathcal{D}$ everywhere except a discrete set of points which are poles of $F$, i.e. Laurent's series of $F$ at such a point has at most a finite number of negative terms, then $F$ is said to be meromorphic.

We introduce some notations. Let $\mathcal{L}(X, Y)$ be the Banach space of bounded linear operators from a Banach space $X$ into a Banach space $Y$. We will use the symbol $\mathcal{H}(X)$ for the class of functions with values in $X$ which are holomorphic with respect to $m \in \mathcal{D}$, and $\mathcal{M}(X)$ for the class of meromorphic on $m \in \mathcal{D}$ functions with values in $X$. For the sake of brevity we also introduce the notations $\mathcal{H}(X, Y):=$ $\mathcal{H}(\mathcal{L}(X, Y)), \mathcal{M}(X, Y):=\mathcal{M}(\mathcal{L}(X, Y))$.

We will treat the problem (3.1) by the technique introduced in [SP, Sec. XVI.4]. Let $R$ be a fixed number larger than $a_{n}, \Pi_{R}:=$ $\Pi \cap\left\{x: x_{1}<R\right\}$, and $g$ a function from $L^{2}\left(\Pi_{R}\right)$ which can be also regarded as an element of $L^{2}(\Pi)$ if we put it equal to zero for $x_{1}>R$. The problem

$$
(\Delta+1) v=m^{2} v+g, \quad x \in \Pi, \quad v=0, \quad x \in \partial \Pi,
$$

can be easily solved by separation of variables, the solution being

$$
\begin{aligned}
v(x, m) & =-\sum_{k=1}^{\infty} \frac{1}{\pi M_{k}} \int_{\Pi} G_{k}(x, t, m) g(t) d^{2} t, \\
G_{k}(x, t, m) & =\left(\mathrm{e}^{-M_{k}\left|x_{1}-t_{1}\right|}-\mathrm{e}^{-M_{k}\left(x_{1}+t_{1}\right)}\right) \sin k t_{2} \sin k x_{2},
\end{aligned}
$$


where $M_{1}=m$ and $M_{k}=\sqrt{k^{2}-1+m^{2}}$ for $k \geq 2$. For the sake of brevity we use the notation $d^{2} t=d t_{1} d t_{2}$. Obviously, the formula (3.4) is valid for all $m \in \mathcal{D}$, not only for $\operatorname{Re} m>0$. The function $v$ can be represented as $v=T_{1}(m) g$, where $T_{1}(m): L^{2}\left(\Pi_{R}\right) \rightarrow H^{2}\left(\Pi_{\widetilde{R}}\right)$ is a bounded linear operator for any positive $\widetilde{R}$. It is straightforward to check that $v \in \mathcal{H}\left(H^{2}\left(\Pi_{\widetilde{R}}\right)\right)$ and $T_{1}(\cdot) \in \mathcal{H}\left(L^{2}\left(\Pi_{R}\right), H^{2}\left(\Pi_{\widetilde{R}}\right)\right)$ in $\mathcal{D}$ for any positive $\widetilde{R}$.

In the next step we consider another boundary value problem for an unknown function $w$, namely

$$
\begin{aligned}
\Delta w & =\Delta v, \quad x \in \Pi_{R}, \\
w & =0, \quad x \in\left(\Gamma_{+} \cup \Gamma_{0}\right) \cap \partial \Pi_{R}, \\
w & =v, \quad x_{1}=R, \\
\frac{\partial w}{\partial x_{2}} & =0, \quad x \in \gamma_{0}, \\
l_{x} w & =0, \quad x \in \gamma .
\end{aligned}
$$

Since $v \in H^{2}\left(\Pi_{R}\right)$ we have $\Delta v \in L^{2}\left(\Pi_{R}\right)$, and thus the problem (3.5) has a unique solution $w \in H^{1}\left(\Pi_{R}\right)$ - see, e.g., [La, Sec. 2.5, Rem. 5.1]. Using the standard theorem on smoothness of solutions of elliptic boundary value problems we can conclude that $w \in H^{2}\left(\Pi_{R, s}\right)$ holds for each $s>0$, where $\Pi_{R, s}:=\Pi_{R} \backslash D_{s}$ with $D_{s}:=\left\{x: x_{2}>0\right.$, $\left.\left(x_{1}-a_{n}\right)^{2}+x_{2}^{2}<s^{2}\right\}$. Hence we can define the linear operator $T_{2}$ by $w=: T_{2} v$ which is a linear bounded map from $H^{2}\left(\Pi_{R}\right)$ into $H^{1}\left(\Pi_{R}\right)$, and from $H^{2}\left(\Pi_{R}\right)$ into $H^{2}\left(\Pi_{R, s}\right)$ for each fixed $s$.

Let $\chi_{R}\left(x_{1}\right)$ be an infinitely differentiable mollifier function equal to one for $x_{1} \leq R-1$ and vanishing for $x_{1} \geq R$. We take the two functions considered above and construct $u$ as a smooth interpolation between them,

$$
u(x, m):=\chi_{R}\left(x_{1}\right) w(x, m)+\left(1-\chi_{R}\left(x_{1}\right)\right) v(x, m) .
$$

Since $w(x, m)=T_{2} T_{1}(m) g$, we have $w \in \mathcal{H}\left(H^{1}\left(\Pi_{R}\right)\right) \cap \mathcal{H}\left(H^{2}\left(\Pi_{R, s}\right)\right)$ as a function of $m$ for each $s>0$. Thus we can introduce the operator $T_{3}(m)$ which maps a function $g \in L^{2}\left(\Pi_{R}\right)$ to the function $u$ determined by (3.6), where $v$ and $w$ are the solutions of (3.3) and (3.5), 
respectively, for this $g$. It easy to see that $T_{3}(\cdot) \in \mathcal{H}\left(L^{2}\left(\Pi_{R}\right), H^{1}\left(\Pi_{\widetilde{R}}\right)\right)$ and $T_{3}(\cdot) \in \mathcal{H}\left(L^{2}\left(\Pi_{R}\right), H^{2}\left(\Pi_{\widetilde{R}, s}\right)\right)$ in $\mathcal{D}$ for any pair of positive $\widetilde{R}, s$.

According to the definition of $v$ and $w$, the function $u$ satisfies all the boundary condition of (3.1). Applying the operator $\left(\Delta+1-m^{2}\right)$ to this function, we obtain

$$
\begin{aligned}
\left(\Delta+1-m^{2}\right) u & =g+(w-v)\left(\Delta+1-m^{2}\right) \chi_{R}+ \\
& +2\left(\nabla_{x} \chi_{R}, \nabla_{x}(w-v)\right)_{\mathbb{R}^{2}}
\end{aligned}
$$

where we have used in the calculation the equations which $v$ and $w$ satisfy. This result shows that the function $u$ solves the problem (3.1) if and only if $g$ satisfies the following equation,

$$
g+T_{4}(m) g=f,
$$

where

$$
T_{4}(m) g:=(w-v)\left(\Delta+1-m^{2}\right) \chi_{R}+2\left(\nabla_{x} \chi_{R}, \nabla_{x}(w-v)\right)_{\mathbb{R}^{2}} ;
$$

recall that both $w$ and $v$ are obtained from $g$ by actions of the operators specified above.

Proposition 3.1 The operator $T_{4}(m)$ is compact for any $R>0$ as an element of $\mathcal{L}\left(L^{2}\left(\Pi_{R}\right), L^{2}\left(\Pi_{R}\right)\right)$ and the function $m \mapsto T_{4}(m)$ belongs to $\mathcal{H}\left(L^{2}\left(\Pi_{R}\right), L^{2}\left(\Pi_{R}\right)\right)$ in $\mathcal{D}$.

Proof: We denote

$$
\begin{aligned}
& T_{41}(m) g:=(w-v)\left(\Delta+1-m^{2}\right) \chi_{R}, \\
& T_{42}(m) g:=2\left(\nabla_{x} \chi_{R}, \nabla_{x}(w-v)\right)_{\mathbb{R}^{2}}+(w-v) \Delta \chi_{R} .
\end{aligned}
$$

Using the described properties of $T_{1}(m)$ and $T_{2}$ it is easy to see that $T_{41}(m)$ is a bounded linear map from $L^{2}\left(\Pi_{R}\right)$ into $H^{1}\left(\Pi_{R}\right)$. The operator-valued function $T_{41}(\cdot)$ belongs to $\mathcal{H}\left(L^{2}\left(\Pi_{R}\right), H^{1}\left(\Pi_{R}\right)\right)$. The function $\chi_{R}^{\prime}$ is smooth and its support lies in $\Pi_{R} \backslash \bar{\Pi}_{R-1}$. Using this together with the fact that the function $w-v$ belongs to $H^{2}\left(\Pi_{R} \backslash \bar{\Pi}_{R-1}\right)$, we conclude that $T_{42}(m): L^{2}\left(\Pi_{R}\right) \rightarrow H^{1}\left(\Pi_{R}\right)$ is a linear bounded operator belonging to $\mathcal{H}\left(L^{2}\left(\Pi_{R}\right), H^{1}\left(\Pi_{R}\right)\right)$ as a function of $m$. Hence 
$T_{4}(\cdot)=T_{41}(\cdot)+T_{42}(\cdot) \in \mathcal{H}\left(L^{2}\left(\Pi_{R}\right), H^{1}\left(\Pi_{R}\right)\right)$, and therefore $T_{4}(m)$ is compact when considered as an operator from $L^{2}\left(\Pi_{R}\right)$ to $L^{2}\left(\Pi_{R}\right)$, belonging to $\mathcal{H}\left(L^{2}\left(\Pi_{R}\right), L^{2}\left(\Pi_{R}\right)\right)$ w.r.t. $m$.

Proposition 3.1 shows that the equation (3.7) can be studied using Fredholm theorems. This will help us to solve our original problem: to construct the solution of (3.1) we have to solve the equation (3.7), then by the procedure described above its solution gives rise to the solution $u$ of the boundary value problem (3.1): $u=T_{3}(m) g$.

Proposition 3.2 The problems (3.7) and (3.1) are equivalent: to each solution $g$ of (3.7) there is a unique solution $u=T_{3}(m) g$ of (3.1), and vice versa, for each solution of (3.1) there exists a unique $g$ solving (3.X) such that $u=T_{3}(m) g$. The equivalence holds for any $m \in \mathcal{D}$.

Proof: The first part has been proved above, it remains to check invertibility of the operator $T_{3}$. Let $u$ be a solution of the problem (3.1). Notice that the function $u$ is infinitely differentiable outside the support of $f$ since there it is a solution of a homogeneous equation. We have to construct the solution $g$ of (3.7) such that $u=T_{3} g$. Let us first determine the functions $v$ and $w$. By $U$ we denote a solution of the following problem,

$$
\begin{aligned}
\Delta U & =0, & & x \in \Pi_{R}, \\
U & =0, & & x \in \partial \Pi_{R} \backslash \overline{\gamma_{0} \cup \gamma}, \\
U & =u, & & x \in \gamma_{0} \cup \gamma,
\end{aligned}
$$

which is unique in $H^{1}\left(\Pi_{R}\right)$, and moreover, it belongs to $C^{\infty}\left(\Pi_{R}\right)$ by a standard result on the smoothness of solutions of elliptic equations. We set

$$
\begin{aligned}
v(x, m) & :=u(x, m)-\chi_{R}\left(x_{1}\right) U(x, m), \\
w(x, m) & :=u(x, m)+\left(1-\chi_{R}\left(x_{1}\right)\right) U(x, m) .
\end{aligned}
$$

One checks easily that the functions $u=\chi_{R} w+\left(1-\chi_{R}\right) v, w$ and $v$ satisfy all the required boundary condition and that $\Delta w=\Delta v$ holds 
in $\Pi_{R}$. Now it suffices to use the equation of the problem (3.3) to determine the function $g$ by

$$
\begin{aligned}
g(x, m) & :=\left(\Delta+1-m^{2}\right) v(x, m) \\
& =\left(\Delta+1-m^{2}\right)\left(u(x, m)-\chi_{R}\left(x_{1}\right) U(x, m)\right) \\
& =f(x)-\left(\Delta+1-m^{2}\right)\left(\chi_{R}\left(x_{1}\right) U(x, m)\right) .
\end{aligned}
$$

Let us check that this $g$ solves (3.7). Using the definition of $U, v$, and $w$, we compute directly the action of $T_{4}(m)$ on $g$ obtaining

$$
\begin{aligned}
T_{4}(m) g & =U\left(\Delta+1-m^{2}\right) \chi_{R}+2\left(\nabla_{x} \chi_{R}, \nabla_{x} U\right) \\
& =U\left(\Delta+1-m^{2}\right) \chi_{R}+2\left(\nabla_{x} \chi_{R}, \nabla_{x} U\right)+\chi_{R} \Delta U \\
& =\left(\Delta+1-m^{2}\right)\left(\chi_{R} U\right) .
\end{aligned}
$$

The last two relations show that $g$ is a solution of the equation (3.7). Let us check the uniqueness: suppose that there are two solutions $g_{1}$ and $g_{2}$ of (3.7) leading to the same function $u^{(1)}=u^{(2)}$. Then $g:=g_{1}-g_{2} \neq 0$ gives rise to $u:=u^{(1)}-u^{(2)}=0$. Let $v$ and $w$ be the solutions of (3.3) and (3.5), respectively, associated with $u$, and put $U:=w-v$. Then it easy to see that this $U$ solves (3.8), where the boundary condition on $\gamma_{0} \cup \gamma$ is homogeneous. Such a solution of (3.8) is unique, $U=0$. Thus $w=v=u=0$ holds in $\Pi_{R}$, and therefore $g=\left(\Delta+1-m^{2}\right) v=0$ in $\Pi_{R}$, which is a contradiction.

The solution of eq. (3.7) depends on $m$, i.e. $g=g(x, m)$. Our next aim is to clarify a nature of this dependence and to look what this implies for the solution of the problem (3.1). We employ the following result borrowed from [SP, Sec. XV.7]:

Theorem 3.3 Let $\mathcal{D}$ be an open connected domain of the complex plane of the variable $m$ and $\{T(m): m \in \mathcal{D}\}$ be a bounded holomorphic family of compact operators from the Banach space $X$ into itself. Moreover, assume that there exists a point $m_{*} \in D$ such that $\left(I+T\left(m_{*}\right)\right)^{-1} \in \mathcal{L}(X, X)$. Then $m \mapsto(I+T(m))^{-1}$ is a meromorphic function in $\mathcal{D}$ with values in $\mathcal{L}(X, X)$.

In our case the Banach space mentioned in Theorem 3.3 is $L^{2}\left(\Pi_{R}\right)$ and $T(m)=T_{4}(m)$. The existence of $m_{*}$ is easy to establish: since the 
equation (3.7) is equivalent to the boundary-value problem (3.1) by Proposition 3.2, it is sufficient to prove the existence of $m_{*}$ for which the problem (3.1) has no nontrivial solution for $f=0$ and $m=m_{*}$ with the asymptotics (3.2). This is true for $m_{*}>0$ which is sufficiently small, because assuming the contrary would lead us to the conclusion that $H_{a_{n}}$ has the eigenvalue $\left(1-m_{*}^{2}\right)$, and this in turn would contradict to the claims (a) and (c) of Proposition 2.1. The compactness of $T_{4}(m)$ and its holomorphic dependence on $m$ follow from Proposition 3.1.

Thus we may apply Theorem 3.3 to the equation (3.7). We implies that $\left(I+T_{4}(m)\right)^{-1}$ exists and it is meromorphic as an operator-valued function in $\mathcal{D}, \quad\left(I+T_{4}(\cdot)\right)^{-1} \in \mathcal{M}\left(L^{2}\left(\Pi_{R}\right), L^{2}\left(\Pi_{R}\right)\right)$. Poles of this function are the values of $m$ for which the equation (3.7) with the vanishing right-hand side has a nontrivial solution. The value $m_{0}:=0$ has this property as it follows from the claim (c) of Proposition 2.1. Let $\phi_{0}$ be a solution of the equation (3.7) for $f=0$ and $m=m_{0}$. The function $\phi_{0}$ is unique up to a multiplicative constant, the uniqueness being implied by Proposition 2.1(c) and Proposition 3.1. The remaining ambiguity is removed if we set $\psi^{(0)}=T_{3}(0) \phi_{0}$. Making the domain $\mathcal{D}$ smaller if necessary we can achieve that zero is the only pole of the function $\left(I+T_{4}(\cdot)\right)^{-1}$ contained in $\mathcal{D}$. In such a case the solution $g=\left(I+T_{4}(m)\right)^{-1}$ of the equation (3.7) can be for any nonzero $m \in \mathcal{D}$ represented as

$$
g=g(m)=\frac{g_{-k}}{m^{k}}+\widetilde{g}(m),
$$

where the integer $k \geq 1$ is the order of the pole, the functions $g_{-k}, \widetilde{g}(m)$ belong to $L^{2}\left(\Pi_{R}\right)$ for nonzero $m \in \mathcal{D}$, and $\widetilde{g}(\cdot) \in \mathcal{M}\left(L^{2}\left(\Pi_{R}\right)\right)$ may have a pole at zero of order not exceeding $k-1$.

Let us stress that Theorem 3.3 says nothing about the order of this pole. Next we are going to prove that the pole in (3.9) is simple, i.e. $k=1$. Substituting (3.9) into (3.7) and comparing the leading terms in $m$ we see that $g_{-k}=K_{0}[f] \phi_{0}$, where $K_{0}[f]$ is a constant depending on $f$. The representation (3.9) in turn yields the following expression for $u$ :

$$
u=T_{3}(m) g=\frac{K_{0}[f] \psi^{(0)}}{m^{k}}+\widetilde{u}(m) .
$$


Here $\widetilde{u}(\cdot)$ is a function belonging to $\mathcal{M}\left(H^{1}\left(\Pi_{\widetilde{R}}\right)\right) \cap \mathcal{M}\left(H^{2}\left(\Pi_{\widetilde{R}, s}\right)\right)$ for any positive $\widetilde{R}, s$ which again may have pole at zero of order not exceeding $k-1$. Multiplying the equation in the problem (3.1) by $\psi^{(0)}$ and integrating by parts we see that

$$
\int_{\Pi_{\tilde{R}}} f \psi^{(0)} d^{2} x=-m^{2} \int_{\Pi_{\tilde{R}}} \psi^{(0)} u d^{2} x+\left.\int_{0}^{\pi}\left(\psi^{(0)} \frac{\partial u}{\partial x_{1}}-u \frac{\partial \psi^{(0)}}{\partial x_{1}}\right)\right|_{x_{1}=\widetilde{R}} d x_{2} .
$$

For $d$ sufficiently large we have

$$
\begin{aligned}
& \left.u\right|_{x_{1}=\widetilde{R}}=\sum_{j=1}^{\infty} C_{j}(m) \sin j x_{2},\left.\quad \psi^{(0)}\right|_{x_{1}=\widetilde{R}}=\sum_{j=1}^{\infty} c_{j} \sin j x_{2}, \\
& \left.\frac{\partial u}{\partial x_{1}}\right|_{x_{1}=\widetilde{R}}=-\sum_{j=1}^{\infty} \sqrt{j^{2}-1+m^{2}} C_{j}(m) \sin j x_{2}, \\
& \left.\frac{\partial \psi^{(0)}}{\partial x_{1}}\right|_{x_{1}=\widetilde{R}}=-\sum_{j=2}^{\infty} c_{j} \sqrt{j^{2}-1} \sin j x_{2},
\end{aligned}
$$

where the functions $C_{j}$ in view of (3.10) behave as

$$
C_{j}(m)=\frac{K_{0}[f] c_{j}}{m^{k}}+\mathcal{O}\left(m^{-k+1}\right) .
$$

Combining the above relations and using the normalization condition $c_{1}=\sqrt{2 / \pi}$ we deduce that

$$
\left.\int_{0}^{\pi}\left(\psi^{(0)} \frac{\partial u}{\partial x_{1}}-u \frac{\partial \psi^{(0)}}{\partial x_{1}}\right)\right|_{x_{1}=\widetilde{R}} d x_{2}=-\frac{K_{0}[f]}{m^{k-1}}+\mathcal{O}\left(m^{-k+2}\right) .
$$

The first integral at the right hand of (3.11) behaves as

$$
-m^{2} \int_{\Pi_{\tilde{R}}} \psi^{(0)} u d^{2} x=-\frac{K_{0}[f]}{m^{k-2}} \int_{\Pi_{\tilde{R}}}\left|\psi^{(0)}\right|^{2} d^{2} x+\mathcal{O}\left(m^{-k+3}\right) .
$$


in the limit $m \rightarrow 0$. Substituting the expressions obtained above into (3.11) and comparing the coefficients at the powers of $m$, we conclude that $k=1$, and furthermore, that

$$
K_{0}[f]=-\int_{\Pi_{\tilde{R}}} f \psi^{(0)} d^{2} x=-\int_{\Pi} f \psi^{(0)} d^{2} x,
$$

To get the last relation we have used the fact that $f$ has a compact support by assumption which therefore lies in $\Pi_{\widetilde{R}}$ for $\widetilde{R}$ sufficient large.

Next we denote $A(m)=T_{3}(m)\left(I+T_{4}(m)\right)^{-1}$. It follows from the relations (3.10), (3.12) that

$$
\begin{aligned}
& u=A(m) f=: A_{0}(m) f+A_{1}(m) f=K_{0}[f] \frac{\psi^{(0)}}{m}+A_{1}(m) f, \\
& A_{1}(\cdot) \in \mathcal{H}\left(L^{2}\left(\Pi_{R}\right), \mathcal{H}\left(H^{1}\left(\Pi_{\widetilde{R}}\right)\right) \cap \mathcal{H}\left(L^{2}\left(\Pi_{R}\right), H^{2}\left(\Pi_{\widetilde{R}, s}\right)\right),\right.
\end{aligned}
$$

where $\widetilde{R}$ and $s$ are arbitrary positive.

It is convenient to summarize all the conclusions made above in a single theorem which represents the main result of this subsection.

Theorem 3.4 Let $f \in L^{2}\left(\Pi_{R}\right)$, and assume that $\widetilde{R}$, $s$ are arbitrary positive numbers, $m \in \mathcal{D}$. Then for $m \in \mathcal{D} \backslash\{0\}$ there exists a unique solution of the boundary value problem (3.1) given by $u=A(m) f$. As a function on $m$ this solution belongs to $\mathcal{M}\left(H^{1}\left(\Pi_{\widetilde{R}}\right)\right) \cap \mathcal{M}\left(H^{2}\left(\Pi_{\widetilde{R}, s}\right)\right)$. The neighborhood $\mathcal{D}$ can be chosen in such a way that the function $A(\cdot) \in \mathcal{M}\left(L^{2}\left(\Pi_{R}\right), H^{1}\left(\Pi_{\widetilde{R}}\right)\right) \cap \mathcal{M}\left(L^{2}\left(\Pi_{R}\right), H^{2}\left(\Pi_{\widetilde{R}, s}\right)\right)$ has just one pole $m_{0}=0$ which is simple. The operator $A(m)$ can be decomposed into the sum $A(m)=A_{0}(m)+A_{1}(m)$, where the operators $A_{i}(m)$ are defined by (3.13). Finally, the relation (3.19) holds true.

\subsection{The asymptotic analysis}

To find the behavior of the quantity $\lambda_{n}(a)$ around the threshold we will analyze $m_{\varepsilon}$ defined as $m_{\varepsilon}^{2}:=1-\lambda_{n}\left(a_{n}+\varepsilon\right)$, which satisfies $m_{\varepsilon} \rightarrow 0$ as $\varepsilon \rightarrow 0$. Let $y=\left(y_{1}, y_{2}\right)$ be the Cartesian coordinates of a point in the strip. The boundary value problem problem for the eigenfunction 
$\psi^{(\varepsilon)}$ can be then written as

$$
\begin{aligned}
\left(\Delta_{y}+1\right) \psi^{(\varepsilon)} & =m_{\varepsilon}^{2} \psi^{(\varepsilon)}, \quad y \in \Pi, \\
l_{y} \psi^{(\varepsilon)} & =0, \quad y \in \gamma, \\
\psi^{(\varepsilon)} & =0, \quad y \in \Gamma_{\varepsilon} \cup \Gamma_{+}, \\
\frac{\partial \psi^{(\varepsilon)}}{\partial y_{2}} & =0, \quad y \in \gamma_{\varepsilon} .
\end{aligned}
$$

As we have announced, we want to get rid of the $\varepsilon$-dependent boundary condition passing to one independent on $\varepsilon$, while the corresponding equation will have coefficients which depend smoothly on $\varepsilon$. To construct the appropriate transformation we use an infinitely differentiable mollifier function $\chi$ of the variable $y_{1}$ which is equal to one for $y_{1} \in\left[\beta_{2}, \beta_{3}\right]$ and vanishes for $y_{1} \leq \beta_{1}$ and $y_{1} \geq \beta_{4}$. Here $\beta_{i}, i=1, \ldots, 4$, are positive constants, $\beta_{1}<\beta_{2}<\beta_{3}<\beta_{4}$, such that $\beta_{2}<a_{n}+\varepsilon<\beta_{3}$ holds for all $\varepsilon$ from a fixed neighborhood of zero. We consider the function $x_{1}: \mathbb{R}_{+} \rightarrow \mathbb{R}_{+}$defined as

$$
x_{1}: x_{1}\left(y_{1}, \varepsilon\right)=y_{1}-\varepsilon \chi\left(y_{1}\right) .
$$

It is easy to see, in particular, that $x_{1}=a_{n}$ as $y_{1}=a_{n}+\varepsilon$. Taking the first two derivatives we get

$$
\frac{d x_{1}}{d y_{1}}=1-\varepsilon \chi^{\prime}\left(y_{1}\right), \quad \frac{d^{2} x_{1}}{d y_{1}^{2}}=-\varepsilon \chi^{\prime \prime}\left(y_{1}\right),
$$

from where it follows that for all sufficiently small $\varepsilon$ the first derivative of $x_{1}$ w.r.t. $y_{1}$ is nonzero everywhere in $\mathbb{R}_{+}$. Thus the map $x_{1}$ is bijective and can be used to define a change of the variable, $y_{1} \mapsto x_{1}\left(y_{1}, \varepsilon\right)$. The problem (3.14) expressed in the variables $x=\left(x_{1}, x_{2}\right), x_{2}:=y_{2}$, becomes

$$
\begin{aligned}
\left(\Delta_{x}+\varepsilon L_{\varepsilon}+1\right) \psi^{(\varepsilon)} & =m_{\varepsilon}^{2} \psi^{(\varepsilon)}, \quad x \in \Pi, \\
l_{x} \psi^{(\varepsilon)} & =0, \quad x \in \gamma, \\
\psi^{(\varepsilon)} & =0, \quad x \in \Gamma_{0} \cup \Gamma_{+}, \\
\frac{\partial \psi^{(\varepsilon)}}{\partial x_{2}} & =0, \quad x \in \gamma_{0} .
\end{aligned}
$$


The operator $L_{\varepsilon}$ appearing in the transformed equation is defined by

$$
\begin{aligned}
& L_{\varepsilon}:=b_{11}\left(x_{1}, \varepsilon\right) \frac{\partial^{2}}{\partial x_{1}^{2}}+b_{1}\left(x_{1}, \varepsilon\right) \frac{\partial}{\partial x_{1}}, \\
& b_{11}\left(x_{1}, \varepsilon\right):=-2 \chi^{\prime}\left(y_{1}\left(x_{1}, \varepsilon\right)\right)+\varepsilon\left(\chi^{\prime}\left(y_{1}\left(x_{1}, \varepsilon\right)\right)\right)^{2}, \\
& b_{1}\left(x_{1}, \varepsilon\right):=-\chi^{\prime \prime}\left(y_{1}\left(x_{1}, \varepsilon\right)\right) .
\end{aligned}
$$

The functions $b_{11}$ and $b_{1}$ are obviously infinitely differentiable, they vanish for $y_{1} \notin\left[\beta_{1}, \beta_{2}\right] \cup\left[\beta_{3}, \beta_{4}\right]$ and satisfy in the limit $\varepsilon \rightarrow 0$ the asymptotic formulae

$$
b_{11}\left(x_{1}, \varepsilon\right)=-2 \chi^{\prime}\left(x_{1}\right)+\mathcal{O}(\varepsilon), \quad b_{1}\left(x_{1}, \varepsilon\right)=-\chi^{\prime \prime}\left(x_{1}\right)+\mathcal{O}(\varepsilon)
$$

uniformly in the variable $x_{1}$.

Now we can proceed to the calculation of $m_{\varepsilon}$ and $\psi^{(\varepsilon)}$. Proposition 2.1 ensures that the eigenfunction and eigenvalue exist. The function $\psi^{(\varepsilon)}$ decays as $\left|x_{1}\right| \rightarrow \infty$ and $\lambda_{n}\left(a_{n}+\varepsilon\right)$ is real. These two facts imply that $m_{\varepsilon}$ is real and positive. It is obvious that $\psi^{(\varepsilon)}$ solves the problem (3.1) for $m=m_{\varepsilon}$ and $f=-\varepsilon L_{\varepsilon} \psi^{(\varepsilon)}$. This allows us to seek for the solution of (3.15) in the form

$$
\psi^{(\varepsilon)}=A\left(m_{\varepsilon}\right) f_{\varepsilon},
$$

where the function $f_{\varepsilon}$ is assumed to an unknown element of $L^{2}\left(\Pi_{R}\right)$ with $R>\beta_{4}$. This may appear strange at a glance, because we know that $f_{\varepsilon}=-\varepsilon L_{\varepsilon} \psi^{(\varepsilon)}$, however, we want to obtain another formula for $f_{\varepsilon}$ not involving $\psi^{(\varepsilon)}$. The function $\psi^{(\varepsilon)}$ defined by (3.17) satisfies all the boundary condition of the problem (3.15). In order to be a solution of (3.15), it is necessary and sufficient for $\psi^{(\varepsilon)}$ to be a solution of the corresponding equation. Substituting into the latter the formula for $\psi^{(\varepsilon)}$, we arrive at the equation for $f_{\varepsilon}$ which reads

$$
\left(I+\varepsilon L_{\varepsilon} A\left(m_{\varepsilon}\right)\right) f_{\varepsilon}=0 .
$$

In view of (3.13) we have

$$
A\left(m_{\varepsilon}\right) f_{\varepsilon}=K_{0}\left[f_{\varepsilon}\right] \frac{\psi^{(0)}}{m_{\varepsilon}}+A_{1}\left(m_{\varepsilon}\right) f_{\varepsilon} .
$$


We substitute this representation for $A\left(m_{\varepsilon}\right) f_{\varepsilon}$ into (3.18) obtaining

$$
f_{\varepsilon}+\frac{\varepsilon}{m_{\varepsilon}} K_{0}\left[f_{\varepsilon}\right] L_{\varepsilon} \psi^{(0)}+\varepsilon L_{\varepsilon} A_{1}\left(m_{\varepsilon}\right) f_{\varepsilon}=0 .
$$

It is clear that $L_{\varepsilon} A_{1}(\cdot) \in \mathcal{H}\left(L^{2}\left(\Pi_{R}\right), L^{2}\left(\Pi_{R}\right)\right)$ and as a function of $(m, \varepsilon)$ the operator $L_{\varepsilon} A_{1}(m)$ is jointly continuous. Thus for sufficiently small $\varepsilon$ the inverse operator $B(m, \varepsilon)=\left(I+\varepsilon L_{\varepsilon} A_{1}(m)\right)^{-1}: L^{2}\left(\Pi_{R}\right) \rightarrow$ $L^{2}\left(\Pi_{R}\right)$ exists and converges to the identity map as $\varepsilon \rightarrow 0$ uniformly in $m$. It is also obvious that $B(\cdot, \varepsilon) \in \mathcal{H}\left(L^{2}\left(\Pi_{R}\right), L^{2}\left(\Pi_{R}\right)\right)$. Applying now $B\left(m_{\varepsilon}, \varepsilon\right)$ to the equation (3.20) we find

$$
f_{\varepsilon}+\frac{\varepsilon}{m_{\varepsilon}} K_{0}\left[f_{\varepsilon}\right] B\left(m_{\varepsilon}, \varepsilon\right) L_{\varepsilon} \psi^{(0)}=0 .
$$

Applying further $K_{0}$ to (3.21), we get one more equation,

$$
K_{0}\left[f_{\varepsilon}\right]+\frac{\varepsilon}{m_{\varepsilon}} K_{0}\left[f_{\varepsilon}\right] K_{0}\left[B\left(m_{\varepsilon}, \varepsilon\right) L_{\varepsilon} \psi^{(0)}\right]=0
$$

Notice that $K_{0}\left[f_{\varepsilon}\right]$ can not be zero, because otherwise (3.21) would imply $f_{\varepsilon}=0$ which yields $\psi^{(\varepsilon)}=0$. The last equation induces the relation

$$
m_{\varepsilon}=-\varepsilon K_{0}\left[B\left(m_{\varepsilon}, \varepsilon\right) L_{\varepsilon} \psi^{(0)}\right]
$$

which can be regarded as an equation for $m_{\varepsilon}$. It is easy to see that $B(\cdot, \varepsilon) L_{\varepsilon} \psi^{(0)} \in \mathcal{H}\left(L^{2}\left(\Pi_{R}\right)\right)$ and the function $(m, \varepsilon) \mapsto B(m, \varepsilon) L_{\varepsilon} \psi^{(0)}$ is jointly continuous. This immediately implies that $K_{0}\left[B(m, \varepsilon) L_{\varepsilon} \psi^{(0)}\right]$ is a holomorphic with respect to $m$ and jointly continuous as a function of $(m, \varepsilon)$. Consequently, for all $\varepsilon$ small enough the estimate

$$
\varepsilon \mid\left[K_{0}\left[B(m, \varepsilon) L_{\varepsilon} \psi^{(0)}\right]|<| m \mid, \quad m \in \partial \mathcal{D},\right.
$$

holds true. Using this inequality in combination with the Rouché theorem we conclude that the functions $h_{1}: h_{1}(m)=m$ and $h_{2}: h_{2}(m)=$ $m+\left[K_{0}\left[B(m, \varepsilon) L_{\varepsilon} \psi^{(0)}\right]\right.$ have the same numbers of zeroes inside $\mathcal{D}$. This means that the equation (3.22) has a unique solution in $\mathcal{D}$ for all sufficient small $\varepsilon$. On the other hand, due to Proposition 2.1 we know that there is a root of equation (3.22) that converges to zero, namely

$$
m_{\varepsilon}=\sqrt{\lambda_{n}\left(a_{n}+\varepsilon\right)-1}>0 .
$$


Consequently, it is the only root of (3.22). Thus the function

$$
f_{\varepsilon}=-\varepsilon B\left(m_{\varepsilon}, \varepsilon\right) L_{\varepsilon} \psi^{(0)},
$$

where $m_{\varepsilon}$ is the solution of (3.22), solves the equation (3.18). It means that the function $f_{\varepsilon}$ defined by (3.23) gives rise to the eigenfunction $\psi^{(\varepsilon)}=A\left(m_{\varepsilon}\right) f_{\varepsilon}$ corresponding to the eigenvalue $\lambda_{n}\left(a_{n}+\varepsilon\right)=1-m_{\varepsilon}^{2}$. In fact, we have also proved that there is just one value of $m=m_{\varepsilon}$ tending to zero as $\varepsilon \rightarrow 0$, for which the boundary value problem (3.14) has a nontrivial solution. This solution decays as $\left|x_{1}\right| \rightarrow \infty$, i.e. there are no non-decaying or even increasing solutions.

The equation (3.22) allows us to calculate the asymptotic expansion for $m_{\varepsilon}$. Since $B(m, \varepsilon) L_{\varepsilon} \psi^{(0)}$ is holomorphic with respect to $m$ and jointly continuous in $(m, \varepsilon)$, and since $m_{\varepsilon}$ tends to zero as $\varepsilon \rightarrow 0$, then by the equation (3.22) we have

$$
\begin{aligned}
m_{\varepsilon} & =\varepsilon \mu+O\left(\varepsilon m_{\varepsilon}\right), \quad \varepsilon \rightarrow 0, \\
\mu & =-K_{0}\left[L_{0} \psi^{(0)}\right], \\
L_{0} & =-2 \chi^{\prime}\left(x_{1}\right) \frac{\partial^{2}}{\partial x_{1}^{2}}-\chi^{\prime \prime}\left(x_{1}\right) \frac{\partial}{\partial x_{1}} .
\end{aligned}
$$

Combining these relations, we can rewrite the error term as follows,

$$
m_{\varepsilon}=\varepsilon \mu+O\left(\varepsilon^{2}\right), \quad \varepsilon \rightarrow 0 .
$$

Next we want to express the coefficient $\mu$ :

$$
\mu=-\int_{\Pi} \psi^{(0)}\left(2 \chi^{\prime}\left(x_{1}\right) \frac{\partial^{2}}{\partial x_{1}^{2}}+\chi^{\prime \prime}\left(x_{1}\right) \frac{\partial}{\partial x_{1}}\right) \psi^{(0)} d^{2} x .
$$

Using the equation which the function $\psi^{(0)}$ satisfies we find

$$
\left(2 \chi^{\prime}\left(x_{1}\right) \frac{\partial^{2}}{\partial x_{1}^{2}}+\chi^{\prime \prime}\left(y_{1}\right) \frac{\partial}{\partial x_{1}}\right) \psi^{(0)}=(\Delta+1)\left(\chi\left(x_{1}\right) \frac{\partial \psi^{(0)}}{\partial x_{1}}\right) .
$$

Integrating then by parts and taking into account properties of $\psi^{(0)}$ 
together with the definition of the mollifier $\chi$ we have

$$
\begin{aligned}
\mu & =-\lim _{s \rightarrow 0} \int_{\Pi \backslash D_{s}} \psi^{(0)}(\Delta+1)\left(\chi\left(x_{1}\right) \frac{\partial \psi^{(0)}}{\partial x_{1}}\right) d^{2} x= \\
& =\lim _{s \rightarrow 0} \int_{\partial D_{s}}\left(\psi^{(0)} \frac{\partial^{2} \psi^{(0)}}{\partial r \partial x_{1}}-\frac{\partial \psi^{(0)}}{\partial x_{1}} \frac{\partial \psi^{(0)}}{\partial r}\right) d s .
\end{aligned}
$$

In order to evaluate the last integral along $\partial D_{s}$ we replace $\psi^{(0)}$ by its asymptotics (2.5) and pass to limit $s \rightarrow 0$ obtaining

$$
\mu=\frac{1}{2} \alpha^{2} \int_{0}^{\pi} \sin ^{2} \frac{\theta_{+}}{2} d \theta_{+}=\frac{\pi \alpha^{2}}{4}
$$

which proves (2.8) with $\mu=\mu_{n}$. In the same way we get

$$
\begin{aligned}
0 & =\int_{\Pi} x_{1} \frac{\partial \psi_{n}^{(0)}}{\partial x_{1}}(\Delta+1) \psi_{n}^{(0)} d^{2} x=a_{n} \frac{\pi \alpha^{2}}{4}+2 \int_{\Pi} \psi_{n}^{(0)} \frac{\partial^{2} \psi_{n}^{(0)}}{\partial x_{1}^{2}} d^{2} x= \\
& =a_{n} \mu_{n}-2 \int_{\Pi}\left|\frac{\partial \psi_{n}^{(0)}}{\partial x_{1}}\right|^{2} d^{2} x,
\end{aligned}
$$

which yields the representation (2.7) for $\mu$,

$$
\mu_{n}=\frac{2}{a_{n}} \int_{\Pi}\left|\frac{\partial \psi_{n}^{(0)}}{\partial x_{1}}\right|^{2} d^{2} x=\frac{1}{a_{n}} \int_{\Sigma}\left|\frac{\partial \psi_{n}^{(0)}}{\partial x_{1}}\right|^{2} d^{2} x
$$

In the last relation we employed the fact that $\psi_{n}^{(0)}$ has a definite parity.

Let us finally pass to discussion of the eigenfunction. In view of (3.19), (3.23) we find that $\psi^{(\varepsilon)}$ is equal to

$$
A\left(m_{\varepsilon}\right) f_{\varepsilon}=-\frac{\varepsilon K_{0}\left[B\left(m_{\varepsilon}, \varepsilon\right) L_{\varepsilon} \psi^{(0)}\right]}{m_{\varepsilon}} \psi^{(0)}-\varepsilon A_{1}\left(m_{\varepsilon}\right) B\left(m_{\varepsilon}, \varepsilon\right) L_{\varepsilon} \psi^{(0)}
$$


Using now the equation (3.22) together with the fact that the function $A_{1}(m) B(m, \varepsilon) L_{\varepsilon} \psi^{(0)}$ is holomorphic and continuous as a function of the respective variables, we conclude that the relation

$$
\psi^{(\varepsilon)}=\psi^{(0)}-\varepsilon A_{1}\left(m_{\varepsilon}\right) B\left(m_{\varepsilon}, \varepsilon\right) L_{\varepsilon} \psi^{(0)}
$$

is valid in $H^{1}\left(\Pi_{R}\right)$ for each $R$. This yields the relation (2.9) and the asymptotic behavior (2.10) concluding thus the proof of Theorem 2.3.

\section{Acknowledgment}

D.B. is grateful for the hospitality extended to him at NPI AS where a part of this work was done. The research has been partially supported by GA AS under the contract \#1048101. The first and the third authors were partially supported by Russian Fund of Basic Research - Grants 00-15-96038, 02-01-00693 (D.B.) and 02-01-00768 (R.G.) as well as by the Ministry of Education of the Russian Federation - Grant E00-1.0-53.

\section{References}

[BGRS] W. Bulla, F. Gesztesy, W. Renger, B. Simon: "Weakly coupled bound states in quantum waveguides", Proc. Amer. Math. Soc. 127, 1487-1495 (1997).

[Da] E. B. Davies: Spectral theory and differential operators, Cambridge Univ. Press, Cambridge 1995.

[DK] J. Dittrich, J. Kř́̌ž: "Bound states in straight quantum waveguide with combined boundary condidtion", J. Math. Phys. 43 (2002), to appear; ; mp_arc 01-458.

[EK] P. Exner, D. Krejčiř́k: "Waveguides coupled through a semitransparent barrier: a Birman-Schwinger analysis", Rev. Math. Phys. 13, 307-334 (2001).

[EŠTV] P. Exner, P. Šeba, M. Tater, D. Vaněk: "Bound states and scattering in quantum waveguides coupled laterally through a boundary window", J. Math. Phys. 37, 4867-4887 (1996). 
[EV] P. Exner, S.A. Vugalter: "Asymptotic estimates for bound states in quantum waveguides coupled laterally through a narrow window", Ann. Inst. H. Poincaré: Phys. théor. 65, 109-123 (1996).

[Ga1] R.R. Gadyl'shin: "Surface potentials and the method of matching asymptotic expansions in the problem of the Helmholtz resonator", Algebra i Analiz 4, 88-115 (1992); English transl. St. Peterburgs Math. J. 4, 273-296 (1993).

[Ga2] R.R. Gadyl'shin: "On local perturbations of Schrödinger operator in axis", Teoreticheskaya i matematicheskaya fizika, accepted for publication (in Russian); math-ph/0202015, mp_arc 02-65.

[Il] A.M. Il'in: Matching of Asymptotic Expansions of Solutions of Boundary Value Problems, Nauka, Moscow 1989; English transl., Amer. Mat. Soc., Providence, RI, 1992.

[KS] M. Klaus, B. Simon: "Coupling constant thresholds in nonrelativistic quantum mechanics. I. Short-range two-body case", Ann. Phys. 130, 251-281 (1980).

[Po] I.Yu. Popov: "Asymptotics for bound state for laterally coupled waveguides", Rep. Math. Phys. 43, 427-437 (1999).

[NP] Nazarov S.A., Plamenevskij B.A. : Elliptic Problems in Domains with Piecewise Smooth Boundaries, Nauka, Moscow 1991; English transl. Walter de Gruyter, Inc. 1994.

[La] Ladyzhenskaya O.A. : The Boundary Value Problems of Mathematical Physics, Nauka, Moscow 1973; English transl. SpringerVerlag, New York 1985.

[SP] E. Sanchez-Palencia: Non-homogeneous Media and Vibration Theory, Springer-Verlag, New York 1980. 